\title{
Epidural analgesia and perineal tears in diabetic parturients: a retrospective cohort study
}

\begin{abstract}
Introduction: The primary aim of this study was to determine whether the use of epidural analgesia in primiparous diabetic patients undergoing vaginal delivery is associated with a reduction in perineal tears. The secondary aim was to determine whether epidural use is associated with higher rates of post-delivery complications such as neonatal resuscitation.

Patients and methods: This was a retrospective cohort study of all primiparous patients with diabetes having vaginal deliveries during the year of 2014 in the state of New South Wales (NSW), Australia. Patients were grouped into those receiving epidural analgesia and those without epidural analgesia (control) during labour. The outcomes assessed were incidence and severity of perineal tears, incidence of post-partum haemorrhage, neonatal resuscitation and admission rate to the neonatal intensive care unit. Individual variables known to be independent risk factors for perineal tears were controlled for using a stratification analysis.

Results: 996 patients were included in the epidural group and 1110 in the control group. There was no significant difference in the rate of an intact perineum $(\mathrm{OR}=1.04$, $95 \% \mathrm{CI}=0.75$ to $1.44, \mathrm{p}=0.81)$. There was however a significant increase in severe tears $(\mathrm{OR}=2.64,95 \% \mathrm{CI}=1.83$ to $3.81, \mathrm{p}<0.00001)$ and episiotomy $(\mathrm{OR}=2.26,95 \% \mathrm{CI}=1.89-2.71$, $\mathrm{p}<0.00001$ ) with the use of epidural analgesia. There was also a significantly increased rate of requirement for neonatal resuscitation interventions in the epidural group.
\end{abstract}

Conclusion: The present study demonstrates that epidural analgesia use in diabetic patients is associated with greater likelihood of severe tears, episiotomy, or neonatal resuscitation.

Keywords: perineal tear, perineal trauma, epidural, diabetes, labour, analgesia
Volume 8 Issue 3 - 2017

\author{
Baxter D F, 1,2 Melhuish T M,1,2 Sandeford J \\ C, ${ }^{3}$ White L D, ${ }^{1,2}$ Austin J,4 \\ 'Rural Clinical School, The University of New South Wales, \\ Australia \\ ${ }^{2}$ WaggaWagga Rural Referral Hospital,Australia \\ ${ }^{3}$ Royal North Shore Hospital, Australia \\ ${ }^{4}$ Rural Clinical School,The University of Notre Dame,Australia
}

Correspondence: Arcy Baxter, Rural Clinical School, The University of New South Wales, WaggaWagga Rural Referral Hospital,Australia, Email darcy.baxter@unsw.edu.au

Received: June 13,2017 | Published: August 01, 2017

\section{Introduction}

The use of epidural analgesia in labour has been deemed a key risk factor for perineal trauma, ${ }^{1}$ however there has been little published on this relationship within population subgroups. Women with diabetes in pregnancy, both pre-existing and gestational, comprise a group in which further study is key to reducing morbidity and an increasing burden on health resources. Evaluating the impact of various modes of analgesia employed in labour may inform approaches to the optimal intrapartum management of this group of women who present with high risk pregnancies and face higher morbidity in the form of larger infants and higher rates of intervention or instrumental birth. ${ }^{2}$

Epidural analgesia has been associated with increased rates of instrumental delivery, yet again few studies have extended this to analysis of diabetic patients as a subgroup. ${ }^{3}$ Some researchers propose that this increased risk of instrumental delivery with epidural analgesia may reflect not only increased birth weight of infants born to these mothers, but a resultant increase in the "need" or patient request for this mode of analgesia in labour. A population register study of Swedish women demonstrated that the infant's birth weight was a strong predictor of the use of epidural analgesia. ${ }^{4}$ However, the correlation between epidural analgesia and perineal status is likely to be confounded by both higher infant birth weight and the increased likelihood of instrumental intervention during deliveries, which have been identified as independent risk factors for perineal tears. ${ }^{5,6}$

The effect of epidural analgesia on perineal status across a general population of women delivering vaginally is unclear, with some studies demonstrating a small increased risk of perineal tears whilst others cite this as a protective factor. ${ }^{7,8}$ Severe perineal injuries involving skeletal muscle (third and forth degree tears), such as the levatorani, have been proposed as an area in which dense epidural anaesthesia may prove beneficial. ${ }^{9}$ The primary aim of this study was to determine whether the use of epidural analgesia in primiparous diabetic patients undergoing vaginal delivery is associated with a reduction in perineal tears. The secondary aims were to determine whether epidural use is associated with higher rates of post-delivery complications such as postpartum haemorrhage and neonatal resuscitation.

\section{Methods}

With the approval of the Greater Western Human Research Ethics Committee (LNR/16/GWAHS/143), we conducted a retrospective cohort study of all primiparous patients with diabetes mellitus (including both gestational and pre-existing diabetes) having vaginal deliveries during the year of 2014 in the state of New South Wales (NSW), Australia. We collected data for this study from a computerised database collated by the NSW government. All data were de-identified by the database administrators prior to data retrieval.

We analysed patient demographics, delivery centre size and rurality, and outcome data (incidence and degree of perineal tears, incidence of severe perineal tears (either grade 3 or 4 tear) incidence of post-partum haemorrhage, neonatal resuscitation and admission rate to the neonatal intensive care unit).Due to inconsistency of reporting, patient height and weight data were not available. To assess size of delivery centre, we utilised the NSW Health 'Maternity Service Level' at the time of delivery, which accounts for the service availability at maternity units regardless of their location (Supplementary Box 
S1). ${ }^{10}$ To assess location of birth, we used the well-established ASGS Remoteness Areas Classification, 2011 standard. ${ }^{11}$ Exclusion criteria were limited to patients with missing data and patients that progressed to have a caesarean section.

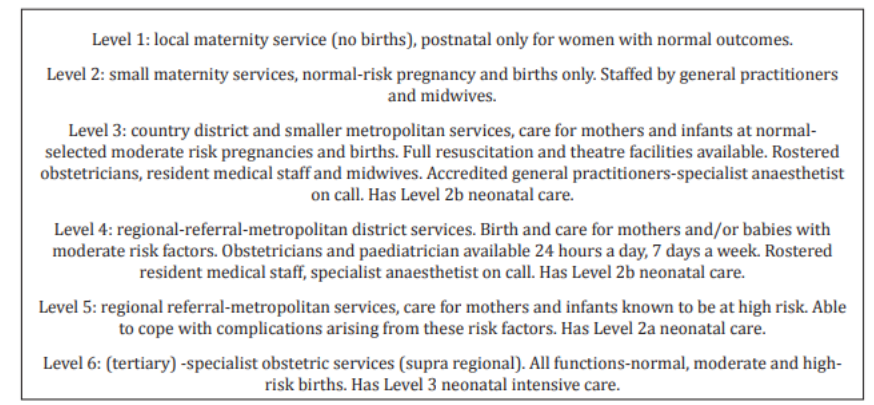

Box SI Maternity service level.

RA1 - Major Cities of Australia

RA2 - Inner Regional Australia

RA3 - Outer Regional Australia

RA4 - Remote Australia

RA5 - Very Remote Australia

\section{Box S2 Remoteness areas classification.}

To control for additional factors influencing the rate of perineal tears and other adverse outcomes, we performed several subgroup analyses. These analyses accounted for patients delivering multiple infants (plurality), those delivering macrosomic neonates $(>4.5$ kilograms) and assisted modes of delivery such as forceps.

\section{Statistical analysis}

Patients with diabetes were grouped into those receiving epidural analgesia and those without epidural analgesia (control) during labour. Outcomes were reported as odds ratios (OR) with a 95\% confidence interval $(95 \% \mathrm{CI})$. Statistical significance was considered to be a two-sided t-test $\mathrm{p}$ value of less than 0.05 for baseline characteristics, and for categorical data such as institutional level the Chi Squared test was used with significance being a $p$ value of less than 0.05 .In assessing multiple outcome measures, we adjusted our alpha level to minimise risk of type 1 errors using the Bonferroni correction method. Given that we assessed 15 outcomes ( 7 primary and 8 secondary) our adjusted significance level for outcomes was $p<0.00333$. Individual variables known to be independent risk factors for perineal tears were controlled for using a stratification analysis. ${ }^{12}$ The statistical analyses were performed through the use of SPSS version 24.0 (SPSS Inc, Chicago, IL).

\section{Results}

During the data collection period, 3194 primiparous diabetic patients had a trial of vaginal delivery. Of these, 996 patients were excluded as they proceeded to a caesarean delivery. Of the remaining patients $(\mathrm{n}=2198), 1088$ received an epidural for analgesia, leaving 1110 patients in the control group. Patient demographics for the two groups are presented in Table 1. It was not possible to control for macrosomia given the very low incidence in this sample. There was a higher incidence of twins in the epidural group $(n=36)$ compared to the control group $(n=2)$. A significantly greater proportion of patients from the control group delivered in smaller level three and four institutions. Conversely, there was a greater proportion of deliveries from the epidural group in private hospitals. There was also significant variation in number of women delivering with and without epidural anesthesia in more rural areas, as indicated by RA classification. A majority of women in RA 1 areas received epidural anaesthesia, whilst in other areas this trend was reversed.

Table I Baseline characteristics

\begin{tabular}{lll}
\hline & $\begin{array}{l}\text { No Epidural } \\
\text { (n= I I I 0) }\end{array}$ & $\begin{array}{l}\text { Epidural } \\
\text { (n= I 088) }\end{array}$ \\
\hline Maternal Factors & & \\
Mean Age (years) & $29+/-5.2$ & $30+/-4.8$ \\
Plurality ( $\geq 2$ babies)* & 2 & 36 \\
Instrumental Delivery* & 202 & 596 \\
Foetal Factors & & \\
Mean Birth Weight (grams) & $3167+/-494.2$ & $3219+/-442.8$ \\
Macrosomia (>4.5kgs) & 4 & 0 \\
Gestational Age (weeks) & $38+/-1.9$ & $39+/-1.5$ \\
Institution Level * & & \\
Level I & $0 \%$ & $0 \%$ \\
Level 2 & $0 \%$ & $0 \%$ \\
Level 3 & $7 \%$ & $2 \%$ \\
Level 4 & $22 \%$ & $12 \%$ \\
Level 5 & $27 \%$ & $23 \%$ \\
Level 6 & $34 \%$ & $37 \%$ \\
Private & $10 \%$ & $26 \%$ \\
Delivery Institution Rurality\#* & \\
RA I & $84.80 \%$ & $9.80 \%$ \\
RA 2 & $11.20 \%$ & $0.40 \%$ \\
RA 3 & $4.10 \%$ & $0 \%$ \\
RA 4 & $0 \%$ & \\
RA 5 & $0 \%$ & \\
\hline
\end{tabular}

*Statistically significant between groups $(p<0.05)$

$\sim$ NSW Health Maternity Institution Level ${ }^{10}$

\#Australia Standard Geographic Classification-Remoteness areas (RA IMajor City; RA 2- Inner Regional; RA 3- Outer Regional; RA 4- Remote; RA 5-Very Remote) ${ }^{\prime \prime}$

Table 2 Perineal status with the use of an epidural after vaginal delivery

\begin{tabular}{lll}
\hline & Odds Ratio (95\% CI) & p Value \\
\hline Intact & $\mathrm{I} .04(0.75-\mathrm{I} .44)$ & $0.8 \mathrm{I}$ \\
Ist degree & $0.55(0.44-0.69)$ & $<0.0000 \mathrm{I}^{*}$ \\
2nd degree & $\mathrm{I} .19(0.96-\mathrm{I} .47)$ & $0.1 \mathrm{I}$ \\
3rd degree & $2.60(\mathrm{I} .78-3.79)$ & $<0.0000 \mathrm{I}^{*}$ \\
4th degree & $2.72(0.68-10.94)$ & 0.16 \\
Severe Tear & $2.64(\mathrm{I} .83-3.8 \mathrm{I})$ & $<0.0000 \mathrm{I}^{*}$ \\
Episiotomy & $2.26(\mathrm{I} .89-2.7 \mathrm{I})$ & $<0.0000 \mathrm{I}^{*}$ \\
\hline
\end{tabular}

$*_{p}<0.00333$

Between the two groups there was no significant difference in the rate of an intact perineum. There was however a significant association between increases in severe tears, and in episiotomy, and the use of epidural analgesia (Table 2). After controlling for instrumental delivery only the increase in episiotomy requirement remained significant (Table 3 ). Controlling for plurality alone, there was a significant increase in the incidence of 2 nd degree tears, as well as severe tears and episiotomies (Table 4). On analysis of secondary outcomes, there was no significant increase in the rate of postpartum haemorrhage or admission to the neonatal intensive care unit. Significantly more neonates required resuscitation interventions in the epidural group. This increase was confined to suction and bag mask ventilation use (Table 5). 
Table 3 Perineal status with the use of an epidural during vaginal delivery adjusted for incidence of instrumental delivery by stratification analysis

\begin{tabular}{lll}
\hline & Odds Ratio $(95 \% \mathbf{C I})$ & p Value \\
\hline Intact & $\mathrm{I} .30(0.89$ to $\mathrm{I} .88)$ & 0.17 \\
Ist degree & $0.64(0.49$ to 0.84$)$ & $0.00 \mathrm{I}^{*}$ \\
2nd degree & $\mathrm{I} . \mathrm{I}(0.9 \mathrm{I}$ to $\mathrm{I} .5 \mathrm{I})$ & $0.2 \mathrm{I}$ \\
3rd degree & $\mathrm{I} .67(\mathrm{I} .00$ to 2.79$)$ & 0.05 \\
4th degree & $2.03(0.28$ to $\mathrm{I} 4.47)$ & 0.48 \\
Severe Tear & $\mathrm{I} .90(\mathrm{I} . \mathrm{I} 5$ to 3.12$)$ & $0.0 \mathrm{I}$ \\
Episiotomy & $\mathrm{I} .53(\mathrm{I} .17$ to $\mathrm{I} .99)$ & $0.002^{*}$ \\
\hline
\end{tabular}

$*_{\mathrm{p}}<0.00333$

Table 4 Perineal status with the use of an epidural during vaginal delivery adjusted for plurality by stratification analysis

\begin{tabular}{lll}
\hline & Odds Ratio (95\%Cl) & p Value \\
\hline Intact & $\mathrm{I} .05(0.75-\mathrm{I} .46)$ & 0.79 \\
I st degree & $0.58(0.46-0.74)$ & $<0.0000 \mathrm{I} *$ \\
2nd degree & $\mathrm{I} .27(\mathrm{I} .03-\mathrm{I} .57)$ & 0.03 \\
3rd degree & $2.63(\mathrm{I} .79-3.85)$ & $<0.0000 \mathrm{I} *$ \\
4th degree & $\mathrm{I} .28(0.75-2.20)$ & 0.37 \\
Severe Tear & $2.68(\mathrm{I} .85-3.88)$ & $<0.0000 \mathrm{I}$ \\
Episiotomy & $2.24(\mathrm{I} .87-2.69)$ & $<0.0000 \mathrm{I}$ \\
\hline
\end{tabular}

$*_{p}<0.00333$

Table 5 Secondary outcomes in patients with $(n=1088)$ and without $(n=1 \mid 10)$ epidural analgesia

\begin{tabular}{|c|c|c|c|c|}
\hline & $\begin{array}{l}\text { No } \\
\text { Epidural }\end{array}$ & Epidural & OR(95\%Cl) & $P$ value \\
\hline $\mathrm{PPH}$ & 23 & 27 & $\mathrm{I} .20(0.69-2 . \mathrm{II})$ & 0.52 \\
\hline \multicolumn{5}{|c|}{ Neonatal resuscitation required } \\
\hline None & 942 & 825 & $0.56(0.45-0.69)$ & $<0.0000 I^{*}$ \\
\hline Suction & 44 & 77 & $1.85(1.26-2.70)$ & $0.002 *$ \\
\hline $\begin{array}{l}\text { Supplemental } \\
\text { Oxygen }\end{array}$ & 36 & 47 & I.35(0.87-2.10) & 0.19 \\
\hline $\begin{array}{l}\text { Bag Valve Mask } \\
\text { Ventilation }\end{array}$ & 78 & 126 & I.76(I.3I-2.36) & $0.0002 *$ \\
\hline Intubation & 9 & 10 & I.I3(0.46-2.80) & 0.78 \\
\hline $\begin{array}{l}\text { External } \\
\text { Cardiac } \\
\text { Massage }\end{array}$ & 2 & 2 & $1.02(0.14-7.26)$ & 0.98 \\
\hline $\begin{array}{l}\text { NICU } \\
\text { Admission }\end{array}$ & $27 \mid$ & 270 & I.02(0.84-I.24) & 0.83 \\
\hline
\end{tabular}

$*_{\mathrm{p}}<0.00333$

\section{Discussion}

In this retrospective cohort study of primiparous patients with diabetes (both gestational and non-gestational) completing vaginal deliveries during the year of 2014, we examined the rates of perineal tears and other adverse outcomes. This is the first study to investigate the outcomes of epidural analgesia during vaginal delivery in diabetic patients. We found that in this subgroup of the population there is an association between the use of epidural analgesia and unfavourable outcomes for both mother and neonate.

Macrosomia has long been an established risk factor for severe perineal tears. Huack et al. ${ }^{13}$ reported an 86 percent increase in the odds of having perineal tear in the context of macrosomia. ${ }^{13}$ Given that diabetes is a significant risk factor for macrosomia, the evidence for each as distinct risk factors for perineal injury has been unclear. ${ }^{14}$ In this study cohort however, it is of note that there were very few macrosomic infants; none in the epidural group and only four in the control group. This may well be because these births were planned or unplanned caesareans and were therefore excluded.

Despite this, the present study demonstrates that epidural use in the presence of diabetes is significantly associated with severe perineal tears $(\mathrm{p}<0.00001)$ and episiotomy $(\mathrm{p}<0.00001)$. This significant effect was maintained when controlling for twin births (plurality); once instrumental delivery was controlled for, the rate of $1^{\text {st }}$ degree tear and episiotomies remained statistically significant.

This suggests that increased incidence of perineal tears and episiotomy use in diabetic patients may be attributed to factors other than macrosomia, instrumental delivery or plurality alone. It is possible that the underlying pathophysiology of diabetes in pregnancy increases the risk of perineal trauma beyond its effect on infant birth weight, via mechanisms such as altered skin and tissue integrity. ${ }^{15}$ The use of epidural was associated with a significant increase in the rate of instrumental delivery. Unlike previous studies however, 1 our results show that even when instrumental delivery is controlled for there is a significantly higher rate of episiotomy. This may be related to a higher general rate of intervention in the labour process.

As others have previously demonstrated, the use of epidural analgesia was lower in smaller or more rural maternity units. ${ }^{16}$ An increased use of epidural analgesia and progression to caesarean section delivery in larger centers may present an inherent bias within the data. It is plausible that higher risk patients receiving epidural analgesia may be more likely to fail a trial of vaginal delivery, thereby increasing the rates of episiotomy and perineal tears observed in this group.

The association between epidural analgesia and adverse outcomes was not confined to maternal outcomes. There was a significantly increased rate of requirement for neonatal resuscitation interventions, namely suction and resuscitative ventilation in the epidural group. There was no related significant increase in the rate of intubation, external cardiac massage or special care nursery (SCN) or neonatal intensive care unit (NICU) admission. This trend is particularly interesting as previous research suggests no difference in neonatal apgar scores (as a surrogate for need for resuscitation) with the use of epidural analgesia. ${ }^{17}$ It would be interesting to assess the use of other modes of analgesia, as one may anticipate a higher use of other modalities including intramuscular opioids to contribute to the need for neonatal stimulation and simple resuscitative measures. The idea that these differences may be due to human factors such as presence of doctor at delivery might correlate with lack of any further need for $\mathrm{SCN} / \mathrm{NICU}$ admission in those same neonates.

There are several limitations of the current retrospective cohort study, with a likely selection bias evident in the number of women in the epidural group excluded after progressing to caesarean section for delivery. As previously highlighted, there were very few infants of birth weight greater than $4500 \mathrm{~g}$ born to women in this study. An indication bias is therefore likely, whereby patients receiving an epidural were inherently higher risk and anticipated to have more complex labour and delivery than those who labored without epidural analgesia.

Data on duration of the second stage of labour were also not available, meaning we were unable to correlate findings with trends in failure to progress in each group. Patients included in the study were classified as diabetic, however there were no further data available on maternal weight or glycaemic control. There were also no specified 
criteria for the diagnosis of diabetes. Although not specific to diabetic women, there is some evidence to support various techniques for protecting against perineal injury in women at risk of tears, such as warm compress or perineal massage..$^{18}$ However, this study was not designed to control for variation among midwifery or medical approaches to perineal management, which may range from "hands off" to episiotomy, and could be further evaluated with a prospective model. ${ }^{19}$

It is also essential to note that this retrospective analysis does not account for the many and varied reasons that epidural analgesia may be provided for women in labour. Whilst it does serve as a highly effective method of analgesia, women perceived to be high risk may also be encouraged to have an epidural placed to facilitate interventions such as assisted delivery should this be clinically indicated.

Future research directions could include a prospective randomised trial of analgesia use in diabetic women, to control for additional confounders and assess causality. Analgesia use in labour and its associations with maternal and neonatal outcomes remains an area with many unknowns and much controversy. In particular, the way in which practice varies by centre size and regional location would be interesting to examine given the significant findings demonstrated here.

In conclusion, options for intrapartum analgesia in diabetic patients are complex and must be considered in the context of increased risk of intervention and potential progression to caesarean section delivery. The likelihood of increased indication for epidural analgesia, particularly with macrosomia, presents a unique challenge to clinicians and patients. This study demonstrates a significant association between an increased rate of perineal tears and use of epidural analgesia in diabetic women delivering for the first time, and increased rate of intervention for the neonate.

\section{Ethical standards}

All patient data were provided in de-identified form by New South Wales Health. This study was approved by local ethics committee and performed in accordance with the Ethical Standards of the 1964 Declaration of Helsinki as revised in 2000.

\section{Acknowledgments}

None.

\section{Conflicts of interest}

The authors have no conflicts of interest to declare.

\section{References}

1. Robinson JN, Norwitz ER, Cohen AP, et al. Epidural Analgesia and Third- or Fourth-Degree Lacerations in Nulliparas. Obstetrics \& Gynecology. 1999;94(2):259-262.

2. Stotland NE, Caughey AB, Breed EM, et al. Risk factors and obstetric complications associated with macrosomia. International Journal of Gynecology and Obstetrics. 2004;87(3):220-226.
3. Anim-Somuah M, Smyth RMD, Jones L. Epidural versus non-epidural or no analgesia in labour. Cochrane Database of Systematic Reviews. 2011;5(5):CD000331.

4. Ekéus C, Hjern A, Hjelmstedt A. The need for epidural analgesia is related to birthweight-a population-based register study. Acta Obstetricia et Gynecologica. 2009;88(4):397-401.

5. Schmitz T, Alberti C, Andriss B, et al. Identification of women at high risk for severe perineal lacerations. European Journal of Obstetrics \& Gynecology and Reproductive Biology. 2014;182:11-15.

6. Groutz A, Hasson J, Wengier A. Third- and fourth-degree perineal tears: prevalence and risk factors in the third millennium. American Journal of Obstetrics and Gynecology. 2011;204(4):347.e1-4.

7. Eskandar O, Shet D. Risk factors for 3rd and 4th degree perineal tear. Journal of Obstetrics and Gynaecology. 29(2):119-122.

8. Groutz A, Hasson J, Wengier A. Third- and fourth-degree perineal tears: prevalence and risk factors in the third millennium. American Journal of Obstetrics and Gynecology. 2011;204(4):347.e1-4.

9. Dietz HP. Pelvic floor trauma in childbirth. Australian and New Zealand Journal of Obstetrics and Gynaecology. 2013;53(3):220-230.

10. NSW Ministry of Health. NSW Maternity and Neonatal Service Capability Framework. Document No. GL2016_018; 2016.

11. Australian Bureau of Statistics. Australian Statistical Geography Standard (ASGS): Volume 5-Remoteness Structure; 2011.

12. Pourhoseingholi, Mohamad Amin, Ahmad Reza Baghestani, et al. How to Control Confounding Effects by Statistical Analysis. Gastroenterology and Hepatology From Bed to Bench. 2012;5(2):79-83.

13. Huack YL, Lewis L, Nathan EA, et al. Risk factors for severe perineal trauma during vaginal childbirth: A Western Australian retrospective cohort study. Women and Birth. 2015;28(1):16-20.

14. Ampt AJ, Ford JB, Roberts CL, et al. Trends in obstetric anal sphincter injuries and associated risk factors for vaginal singleton term births in New South Wales 2001-2009. Australian and New Zealand Journal of Obstetrics and Gynaecology. 2013;53(1):9-16.

15. Bermudez DM, Herdrich BJ, Xu J. Impaired Biomechanical Properties of Diabetic Skin: Implications in Pathogenesis of Diabetic Wound Complications. The American Journal of Pathology. 2011;178(5):22152223.

16. Powers JR, Loxton DJ, O'Mara AT, et al. Regardless of where they give birth, women living in non-metropolitan areas are less likely to have an epidural than their metropolitan counterparts. Women and Birth. 2013;26(2):e77-e81.

17. Lieberman E, O’Donoghue C. Unintended effects of epidural analgesia during labor: A systematic review. American Journal of Obstetrics and Gynecology. 2012;186(5):S31-S68.

18. Aasheim V, Nilsen ABV, Lukasse M, et al. Perineal techniques during the second stage of labour for reducing perineal trauma. Cochrane Database of Systematic Reviews. 2011;12:CD006672.

19. Ampt AJ, de Vroom, M, Ford JB. Perineal management techniques among midwives at five hospitals in New South Wales - A crosssectional survey. Australian and New Zealand Journal of Obstetrics and Gynaecology. 2015;55(3):251-256. 